\title{
Beneficial effects of $\beta$-conglycinin on renal function and nephrin expression in early streptozotocin-induced diabetic nephropathy rats
}

\author{
Hsin-Yi Yang ${ }^{1}$, Lin-Yi Wu ${ }^{2}$, Wan-Ju Yeh ${ }^{2}$ and Jiun-Rong Chen ${ }^{2,3 *}$ \\ ${ }^{1}$ Department of Nutrition, I-Shou University, Kaobsiung 824, Taiwan, ROC \\ ${ }^{2}$ School of Nutrition and Health Sciences, Taipei Medical University, Taipei 110, Taiwan, ROC \\ ${ }^{3}$ Nutrition Research Centre, Taipei Medical University Hospital, Taipei 110, Taiwan, ROC \\ (Submitted 28 September 2012 - Final revision received 8 May 2013 - Accepted 8 May 2013 - First published online 27 June 2013)
}

\begin{abstract}
The objective of the present study was to investigate the effects of $\beta$-conglycinin and soya isoflavones on diabetic nephropathy (DN). DN was induced by an intravenous injection of streptozotocin $(25 \mathrm{mg} / \mathrm{kg})$ in spontaneously hypertensive rats. DN rats were divided into a non-diabetic group (C, control group) and three DN groups (D, DN with control diet; B, DN + control diet with one-eighth of casein replaced by $\beta$-conglycinin as the protein source; and I, DN + control diet with $0 \cdot 01 \%$ soya isoflavones). After a 4-week experimental period, we found that fasting blood sugar and plasma and kidney advanced glycation end product levels and $24 \mathrm{~h}$ urinary protein excretion of the B group were significantly lower than those of the D group and insulin sensitivity and nephrin expression of the B group were significantly higher than those of the D group. In addition, systolic blood pressure, angiotensin-converting enzyme activity, angiotensin II level and plasma TAG level of the B group were significantly lower than those of the D group, whereas only the levels of plasma TAG and thiobarbituric acid-reactive substances of the I group were lower than those of the D group. In conclusion, $\beta$-conglycinin may be beneficial for retarding DN progression and this effect cannot be completely explained by its isoflavone content.
\end{abstract}

Key words: $\beta$-Conglycinin: Soya: Diabetes: Diabetic nephropathy: Hypertension: Nephrin

Diabetic nephropathy (DN) is one of the most serious microvascular complications of diabetes and a major cause of end-stage renal disease ${ }^{(1)}$. Diabetes mellitus (DM) was the fifth cause among the top ten causes of death in Taiwan in 2010. According to the 2004-8 Nutrition and Health Survey in Taiwan (NHST), the prevalence of DM was $9 \cdot 2 \%$, and haemodialysis treatment being required by one-fourth to one-third of the patients is caused by DN.

Systemic hypertension leads to glomerular hypertension, renal microvascular complications and glomerular basement membrane thickening and accelerates renal failure. Renin and angiotensins released from the kidneys during kidney injury lead to elevated blood pressure. These factors may form a vicious cycle and aggravate renal dysfunction. About $50 \%$ of the patients who suffered from type $1 \mathrm{DM}$ for more than 30 years had hypertension ${ }^{(2)}$. The German Society for Diabetes has also suggested that adjusting the blood sugar levels to a near-normal range and normalising the blood pressure are beneficial for preventing DN.
Hyperglycaemia may also lead to haemodynamic changes ${ }^{(3)}$ and excretion of angiotensin II (Ag II) ${ }^{(4)}$. Both hyperglycaemia and elevation of $\mathrm{Ag}$ II levels induce the formation of reactive oxidative species. Exposure of podocytes to high glucose levels leads to an increase in reactive oxidative species generation and is associated with the up-regulation of cytochrome P450 4A (CYP4A) expression, and CYP4A inhibitors can ameliorate proteinuria in $\mathrm{DM}$ mice ${ }^{(5)}$. In addition, nephrin is a podocyte-specific protein and is a component of the filtration slits in the kidneys. In DN, hyperglycaemia and high Ag II levels are related to the dysregulation of nephrin expression and proteinuria $^{(6,7)}$

Modification of dietary habits is important in DM treatment. Soyabean is the main source of dietary plant protein source in Oriental diets. Previous studies have shown that soya protein has several health-promoting effects ${ }^{(8)}$, such as improvement of glucose tolerance, insulin sensitivity and hyperlipidaemia $^{(9)}$ and retardation of the progression of hypertension and renal dysfunction ${ }^{(10)}$. $\beta$-Conglycinin, a major

Abbreviations: ACE, angiotensin-converting enzyme; Ag II, angiotensin II; AGE, advanced glycation end product; B, diabetic nephropathy $+\beta$-conglycinin group; C, control group; Ccr, creatinine clearance rate; CYP4A, cytochrome P450 4A; D, diabetic nephropathy group; DM, diabetes mellitus; DN, diabetic nephropathy; I, diabetic nephropathy + soya isoflavone group; ISI, insulin sensitivity index; SHR, spontaneously hypertensive rats; STZ, streptozotocin; TBARS, thiobarbituric acid-reactive substances; TGF- $\beta$, tumour growth factor- $\beta$.

*Corresponding author: J.-R. Chen, fax +886 22737 3112, email syunei@tmu.edu.tw 
IgG peroxidase-conjugated secondary antibody). Gastrocnemius muscle suspensions were analysed for the expression of PPAR- $\gamma$ (using the polyclonal anti-rabbit PPAR- $\gamma$ antibody, D69, and a goat anti-rabbit IgG peroxidase-conjugated secondary antibody). The immune complex was developed using an enhanced chemiluminescence detection system (Western Lighting ${ }^{\mathrm{TM}}$; PerkinElmer), and bands were quantified by densitometry using Image-Pro Plus 4.5 (Media Cybernetic). Equal loading of the total protein was verified using a commercially available mAb against glyceraldehyde 3-phosphate dehydrogenase, and the results are expressed as the ratio of protein:glyceraldehyde 3-phosphate dehydrogenase.

Pathological analysis. Dissected kidney samples of the rats were fixed in $10 \%$ formaldehyde. The samples were stained with the haematoxylin and eosin stain. Biopsies were examined on a blinded basis by a pathologist, using a 0-3 injury scale, with 0 indicating absent or minimal, 1 indicating mild, 2 indicating moderate and 3 indicating severe injury.

\section{Statistical analysis}

Data were analysed using one-way ANOVA and the least significant difference test using the SAS program (version 9.1; SAS, Inc.). Results are expressed as the mean with their standard errors. A $P$ value $<0.05$ was taken as the level of statistical significance.

\section{Results}

\section{Body weight, food intake and blood pressure}

At the end of the study, body weights of the three diabetic groups (D: 176.1 (SEM 17.6) g; B: 182.6 (SEM 11.2) g; and I: 196.8 (SEM 5.6) g) were lower than that of the $\mathrm{C}$ group (350.0 (sEm 6.5)g) $(P=0.000)$, and food intake of the three diabetic groups (D: 32.3 (SEM 1.2) g; B: 30.4 (SEM 1.8) g; and I: $31 \cdot 1(\operatorname{sem} 2 \cdot 0) \mathrm{g})$ was also higher than that of the $\mathrm{C}$ group
(21.3 (SEM 2.5) g) ( $P=0.003)$. No difference in body weight and food intake was found among the D, B and I groups. The systolic blood pressure of the $\mathrm{B}$ group was significantly lower than that of the $\mathrm{D}$ group $(P=0 \cdot 014)$. There was no difference in diastolic blood pressure among all the groups $(P=0 \cdot 406$; Table 2).

\section{Plasma biochemical analysis}

At the end of the study, the plasma glucose level of the $\mathrm{C}$ group was significantly lower $(P=0.000)$ than those of the DN groups, and the plasma glucose level of the $\mathrm{B}$ group was significantly lower $(P=0.036)$ than that of the D group, while no difference was found between the $\mathrm{D}$ and I groups $(P=0.368)$ (Table 2 ). Plasma insulin levels were significantly lower in the three DN groups than in the $C$ group $(P=0.004)$. The ISI of the D group was also lower than that of the $\mathrm{C}$ group $(P=0.005)$, while the $\mathrm{B}$ group had a significantly higher ISI than the D group $(P=0.042)$ (Table 2$)$. The plasma TAG level of the $\mathrm{D}$ group was higher than that of the $\mathrm{C}$ group $(P=0.025)$. No difference was found in total cholesterol concentrations among all the groups (Table 2).

\section{Advanced glycation end product levels}

Plasma AGE levels of the D and I groups were significantly higher than those of the $\mathrm{C}$ and $\mathrm{B}$ groups $(P=0.005)$. The kidney AGE level of the $D$ group was significantly higher than that of the $\mathrm{C}$ group $(P=0.000)$, and those of both the $\mathrm{B}$ and $\mathrm{I}$ groups were lower than that of the D group $(P=0.003)$ (Table 2$)$.

\section{Renal function markers in the plasma and urine}

Blood urea $\mathrm{N}$ levels of the three DN groups were significantly higher than that of the $\mathrm{C}$ group $(P=0.001)$. The plasma uric acid concentration of the $\mathrm{D}$ group was higher than that of

Table 2. Blood pressure, plasma and kidney biochemical analysis at the end of the study (Mean values with their standard errors)

\begin{tabular}{|c|c|c|c|c|c|c|c|c|}
\hline & \multicolumn{2}{|c|}{$C(n 8)$} & \multicolumn{2}{|c|}{$\mathrm{D}(n 6)$} & \multicolumn{2}{|c|}{$\mathrm{B}(n 10)$} & \multicolumn{2}{|c|}{ I $(n 9)$} \\
\hline & Mean & SEM & Mean & SEM & Mean & SEM & Mean & SEM \\
\hline $\mathrm{SBP}(\mathrm{mmHg})$ & $192 \cdot 4^{c}$ & 3.5 & $179 \cdot 0^{\mathrm{b}}$ & 4.4 & $165 \cdot 4^{\mathrm{a}}$ & $2 \cdot 9$ & $177 \cdot 9^{b}$ & 3.5 \\
\hline $\mathrm{DBP}(\mathrm{mmHg})$ & $128 \cdot 0$ & $7 \cdot 0$ & $122 \cdot 4$ & 5.9 & 114.5 & 5.5 & $113 \cdot 6$ & $8 \cdot 3$ \\
\hline Plasma glucose (mg/l) & $1530^{\mathrm{a}}$ & 81 & $3816^{\mathrm{C}}$ & 141 & $3166^{\mathrm{b}}$ & 159 & $4092^{c}$ & 296 \\
\hline Plasma insulin (g/l) & $0.25^{b}$ & 0.04 & $0.18^{a}$ & 0.01 & $0.17^{\mathrm{a}}$ & 0.01 & $0 \cdot 17^{\mathrm{a}}$ & 0.01 \\
\hline ISI & $0.28^{\mathrm{b}}$ & 0.01 & $0.24^{a}$ & 0.01 & $0 \cdot 26^{\mathrm{b}}$ & 0.01 & $0.24^{a}$ & 0.01 \\
\hline TAG (mg/l) & $315^{a}$ & 29 & $1053^{\mathrm{b}}$ & 555 & $450^{a}$ & 57 & $328^{a}$ & 49 \\
\hline $\mathrm{TC}(\mathrm{mg} / \mathrm{l})$ & 518 & 14 & 680 & 65 & 598 & 30 & 641 & 43 \\
\hline Plasma AGE (g/ml) & $1 \cdot 2^{\mathrm{a}}$ & 0.1 & $1 \cdot 6^{\mathrm{b}}$ & 0.1 & $1 \cdot 3^{\mathrm{a}}$ & 0.0 & $1.4^{\mathrm{b}}$ & 0.1 \\
\hline Kidney AGE (mg/g protein) & $7 \cdot 0^{\mathrm{a}}$ & 0.5 & $11 \cdot 1^{d}$ & 0.5 & $8 \cdot 4^{\mathrm{b}}$ & 0.5 & $9 \cdot 6^{\mathrm{b}}$ & 0.4 \\
\hline Plasma ACE activity (mU/ml) & 103.7 & 4.9 & $108 \cdot 8$ & $6 \cdot 3$ & 94.4 & 3.0 & 98.3 & 1.6 \\
\hline Kidney ACE activity (U/g protein) & $8 \cdot 1^{b}$ & 0.7 & $7 \cdot 3^{\mathrm{b}}$ & 0.3 & $4 \cdot 7^{\mathrm{a}}$ & 0.2 & $7 \cdot 7^{\mathrm{b}}$ & 0.5 \\
\hline Plasma angiotensin II (mg/ml) & $140 \cdot 3^{c}$ & 4.4 & $93 \cdot 6^{\mathrm{b}}$ & $2 \cdot 2$ & $81 \cdot 7^{a}$ & 0.9 & $86 \cdot 0^{\mathrm{a}, \mathrm{b}}$ & 0.6 \\
\hline Kidney angiotensin II (mg/g protein) & $11 \cdot 7^{\mathrm{a}}$ & 0.7 & $14 \cdot 6^{\mathrm{b}}$ & $1 \cdot 1$ & $11 \cdot 4^{\mathrm{a}}$ & 0.3 & $14 \cdot 2^{\mathrm{b}}$ & 0.9 \\
\hline Plasma MDA (mM) & $5 \cdot 5^{\mathrm{a}}$ & 0.2 & $6.4^{\mathrm{b}}$ & 0.6 & $5 \cdot 0^{\mathrm{a}}$ & 0.3 & $5 \cdot 1^{\mathrm{a}}$ & 0.2 \\
\hline Kidney MDA (mmol/g protein) & $626 \cdot 1^{\mathrm{a}}$ & $20 \cdot 8$ & $746 \cdot 7^{b}$ & 35.5 & $629 \cdot 6^{\mathrm{a}}$ & 37.6 & $766 \cdot 0^{\mathrm{b}}$ & $45 \cdot 8$ \\
\hline
\end{tabular}

C, control group; D, diabetic nephropathy (DN) group; B, DN + $\beta$-conglycinin group; I, DN + soya isoflavone group; SBP, systolic blood pressure; DBP, diastolic blood pressure; ISI, insulin sensitivity index; TC, total cholesterol; AGE, advanced glycation end products; ACE, angiotensin-converting enzyme; MDA, malondialdehyde. a,b,c,d Mean values with unlike superscript letters were significantly different $(P<0.05)$. 
Table 3. Renal function markers at the end of the study

(Mean values with their standard errors)

\begin{tabular}{|c|c|c|c|c|c|c|c|c|}
\hline & \multicolumn{2}{|c|}{$C(n 8)$} & \multicolumn{2}{|c|}{$\mathrm{D}(n 6)$} & \multicolumn{2}{|c|}{$\mathrm{B}(n 10)$} & \multicolumn{2}{|c|}{ I (n9) } \\
\hline & Mean & SEM & Mean & SEM & Mean & SEM & Mean & SEM \\
\hline \multicolumn{9}{|l|}{ Plasma } \\
\hline BUN (mg/l) & $198^{\mathrm{a}}$ & $5 \cdot 0$ & $373^{\mathrm{b}}$ & 65 & $326^{\mathrm{b}}$ & 15 & $336^{\mathrm{b}}$ & 27 \\
\hline Creatinine (mg/l) & 4.0 & 0.0 & 4.0 & 1.0 & 4.0 & 0.0 & 4.0 & 0.0 \\
\hline Uric acid (mg/l) & $4.0^{\mathrm{a}}$ & 0.0 & $9 \cdot 0^{b}$ & $2 \cdot 0$ & $5 \cdot 0^{\mathrm{a}}$ & 0.0 & $3.0^{\mathrm{a}}$ & 0.0 \\
\hline \multicolumn{9}{|l|}{ Urine } \\
\hline $24 \mathrm{~h}$ volume $(\mathrm{ml} / \mathrm{d})$ & $5 \cdot 3^{\mathrm{a}}$ & 1.4 & $18 \cdot 8^{\mathrm{b}}$ & 0.5 & $17 \cdot 6^{\mathrm{b}}$ & 1.4 & $14 \cdot 5^{\mathrm{b}}$ & 1.6 \\
\hline Glucose (mg/d) & $0.2^{\mathrm{a}}$ & 0.1 & $848 \cdot 2^{\mathrm{b}}$ & $81 \cdot 3$ & $858 \cdot 3^{\mathrm{b}}$ & $136 \cdot 8$ & $642 \cdot 6^{\mathrm{b}}$ & 117.9 \\
\hline Protein $(\mathrm{mg} / \mathrm{d})$ & $10 \cdot 6^{\mathrm{a}}$ & 0.7 & $21 \cdot 0^{\mathrm{b}}$ & $2 \cdot 0$ & $10 \cdot 9^{\mathrm{a}}$ & 1.4 & $17 \cdot 5^{\mathrm{b}}$ & $2 \cdot 5$ \\
\hline $\operatorname{Ccr}(\mathrm{ml} / \mathrm{min})$ & $1.4^{\mathrm{b}}$ & 0.2 & $1 \cdot 1^{\mathrm{a}}$ & 0.2 & $0.9^{\mathrm{a}}$ & 0.1 & $1 \cdot 1^{\mathrm{a}}$ & 0.1 \\
\hline
\end{tabular}

C, control group; D, diabetic nephropathy (DN) group; B, DN + $\beta$-conglycinin group; I, DN + soya isoflavone group; BUN, blood urea $\mathrm{N}$; Ccr, creatinine clearance rate.

${ }^{\mathrm{a}, \mathrm{b}}$ Mean values with unlike superscript letters were significantly different $(P<0.05)$.

the $\mathrm{C}$ group $(P=0.000)$, while no difference was found between the $\mathrm{C}$ group and the $\mathrm{B}$ and I groups. The $24 \mathrm{~h}$ urine volume and urinary glucose excretion of the three DN groups were significantly higher than those of the $C$ group $(P=0 \cdot 000)$. Urinary protein excretion levels of the $\mathrm{D}$ and I groups were significantly higher than that of the $C$ group $(P=0.002)$, while no difference was found between the $\mathrm{C}$ and $\mathrm{B}$ groups $(P=0.914)$. The $\mathrm{Ccr}$ of the DN groups were

(A)

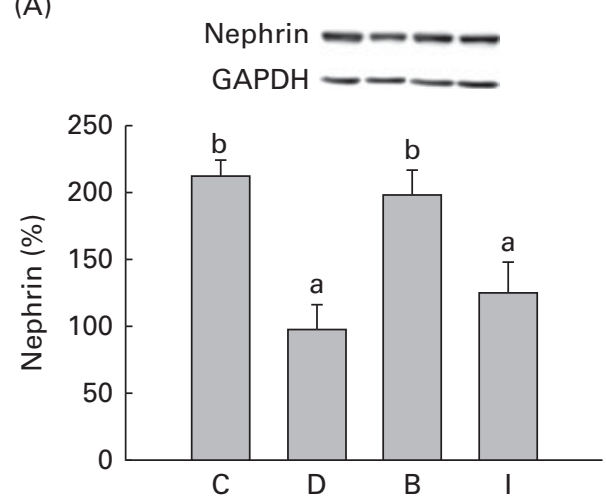

(B)
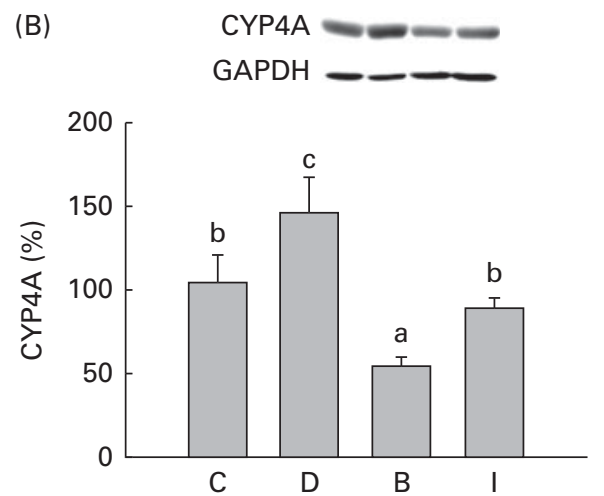

significantly lower than that of the $\mathrm{C}$ group $(P=0 \cdot 018)$, and there was no difference among the three DN groups (Table 3 ).

\section{Angiotensin-converting enzyme activity and angiotensin // level}

At the end of the study, no differences in plasma ACE activity were found among all the groups $(P=0.082)$. However, the kidney ACE activity of the B group was significantly lower

(C)

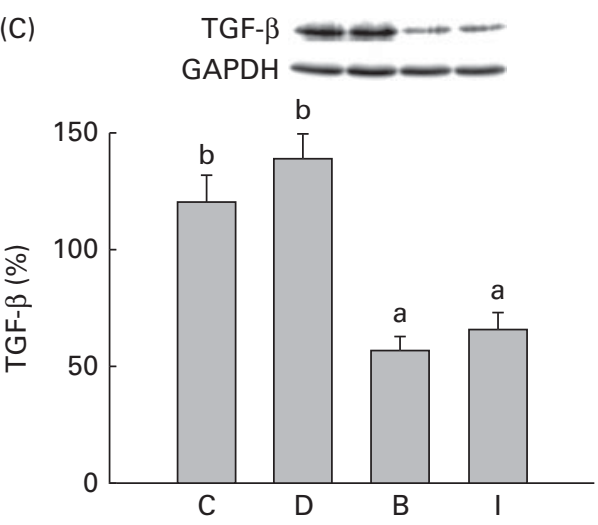

(D)
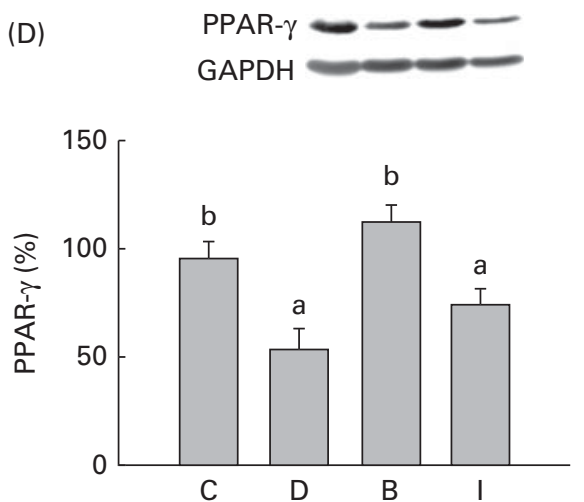

Fig. 1. (A) Nephrin, (B) cytochrome P450 4A (CYP4A) and (C) tumour growth factor (TGF)- $\beta$ protein expressions in the kidneys and (D) PPAR- $\gamma$ protein expression in the gastrocnemius muscle at the end of the study. Values are means, with their standard errors represented by vertical bars. ${ }^{a, b, c}$ Mean values with unlike letters were significantly different $(P<0.05)$. C, control group ( $n$ 8); D, diabetic nephropathy (DN) group $(n 6)$; B, DN $+\beta$-conglycinin group $(n 10) ;$ I, DN + soya isoflavone group $(n 9)$. GAPDH, glyceraldehyde 3-phosphate dehydrogenase. 
than that of the $\mathrm{D}$ group $(P=0 \cdot 000)$, while no difference was found between the D and I groups $(P=0.562)$. Plasma and kidney Ag II concentrations of the B group were significantly lower than those of the D group $(P=0 \cdot 001)$, and there was no difference between the I and D groups $(P=0.738)$ (Table 2$)$.

\section{Nephrin, cytochrome P450 4A and PPAR- $\gamma$ protein expressions}

Nephrin protein expression of the D group was significantly lower than those of the $\mathrm{C}$ and $\mathrm{B}$ groups $(P=0.004)$, and no difference was found between the $\mathrm{I}$ and $\mathrm{D}$ groups $(P=0.148)$ (Fig. 1(A)). CYP4A protein expression of the D group was significantly higher than that of the $C$ group $(P=0.024)$. CYP4A expressions of the $\mathrm{B}$ and I groups were also lower than that of the D group (Fig. 1(B) $(P=0.048)$. Gastrocnemius muscle PPAR- $\gamma$ protein expression of the D group was significantly lower than those of the $\mathrm{C}$ and B groups $(P=0.003)$, but there was no difference between the I and D groups $(P=0 \cdot 119)$ (Fig. 1(D)).

\section{Thiobarbituric acid-reactive substances and tumour growth factor- $\beta$ protein expressions}

Plasma and kidney TBARS levels of the D group were significantly higher than those of the $\mathrm{C}$ group $(P=0.036)$. Plasma TBARS levels of both the $\mathrm{B}$ and I groups were lower than that of the D group $(P=0.002)$, while only the $\mathrm{B}$ group had a lower kidney TBARS concentration than the D group (Table 2). TGF- $\beta$ protein expressions of the $\mathrm{B}$ and I groups were also lower than that of the $\mathrm{D}$ group $(P=0 \cdot 000)$ (Fig. 1(C))

\section{Pathological analysis}

At the end of the study, we found mild renal vessel congestion in the D group. The score of the B group was significantly lower $(P=0.000)$ than that of the $\mathrm{D}$ group, but no significant difference was found between the I and D groups $(P=0 \cdot 050)$ (Fig. 2). No significant glomerulonephritis or interstitial inflammation was observed in all the groups, and the results indicated that our diabetic rats were only undergoing early-stage nephropathy.

\section{Discussion}

In the present study, we treated SHR with a single dose of STZ, and the rats showed typical symptoms of diabetes, such as hyperglycaemia, weight loss, polyuria and glycosuria. In addition, the STZ-treated SHR showed higher blood urea $\mathrm{N}$ and urinary protein excretion and a lower Ccr, characteristics of renal dysfunction. These results indicate that we have successfully established a rat model of early STZ-induced DN. Although all the diabetic rats exhibited increased food intake, no difference was found among the three groups. The B group orally ingested the diet containing $1.75 \%$ $\beta$-conglycinin with $0.01 \%$ soya isoflavones and the D group consumed the same amount of soya isoflavones since both the groups exhibited the same degree of food intake.
(A)
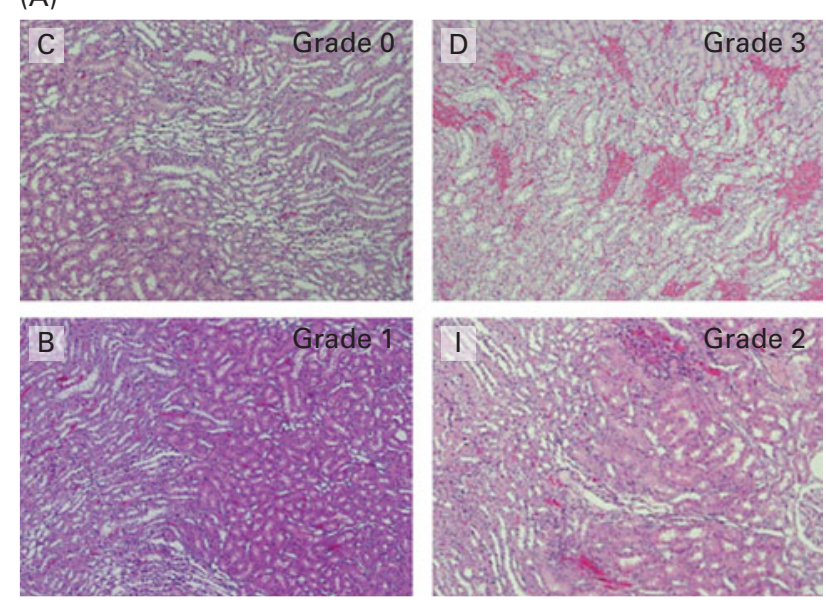

(B)

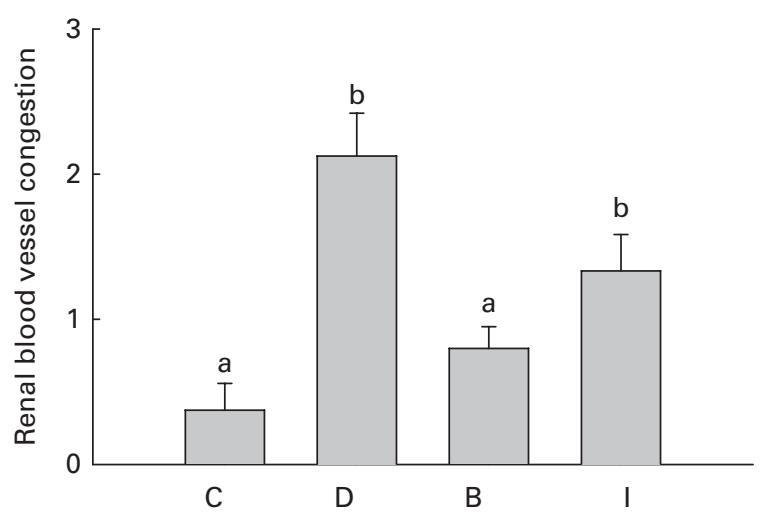

Fig. 2. (A) Histopathology of renal blood vessel congestion (haematoxylin and eosin stain, $40 \times$ ) and (B) score at the end of the study. Values are means, with their standard errors represented by vertical bars. ${ }^{a, b}$ Mean values with unlike letters were significantly different $(P<0.05)$. C, control group ( $n$ 8); D, diabetic nephropathy (DN) group ( $n 6)$; $\mathrm{B}, \mathrm{DN}+\beta$-conglycinin group ( $n 10)$; I, DN + soya isoflavone group ( $n 9)$. Grade 0 , absent or minimal; grade 1, mild; grade 2, moderate; grade 3 , severe. (A colour version of this figure can be found online at http://www.journals.cambridge.org/bjn)

Therefore, we can confirm that the effects $\beta$-conglycinin on diabetic rats are due to its protein composition or soya isoflavones through a comparison of the results of the two groups.

Many studies have reported that soya protein can retard the progression of renal failure ${ }^{(15)}$. $\beta$-Conglycinin is the major storage protein of soyabeans and is considered to be one of the bioactive components in soya protein. In the present study, $\beta$-conglycinin was used to replace one-eighth of casein as the dietary protein, and we found that $\beta$-conglycinin decreased urinary protein excretion. Nephrin is a protein that helps maintain the filtration barrier integrity in the kidneys ${ }^{(16)}$. A human study has indicated that diabetic patients, before the onset of albuminuria, exhibited increased urinary nephrin excretion ${ }^{(17)}$. Thus, nephrin may be an early indicator of renal injury ${ }^{(18)}$. In animal experiments, renal nephrin expression was also lower in diabetic $\mathrm{SHR}^{(19)}$. We found that $\beta$-conglycinin intervention significantly increased nephrin expression and decreased urinary protein excretion in the DN 
rats. However, we did not find changes in nephrin expression or urinary protein excretion in the I group, which consumed the same amount of isoflavones as the $\mathrm{B}$ group. These results suggest that $\beta$-conglycinin supplementation retards renal injury in diabetic rats, and this effect cannot be completely explained by its isoflavone content.

A previous study has reported that $\beta$-conglycinin improves glucose tolerance by increasing insulin sensitivity ${ }^{(20)}$. In the present study, $\beta$-conglycinin decreased the fasting blood glucose levels in the DN rats, but it did not affect plasma insulin concentrations. In addition, we also found lower plasma and kidney AGE concentrations in rats consuming the diets containing $\beta$-conglycinin. AGE can also interrupt renal nephrin expression ${ }^{(6)}$. Therefore, $\beta$-conglycinin might ameliorate hyperglycaemia by increasing insulin sensitivity and thus decrease plasma and kidney AGE levels, which may help to preserve nephrin expression in DN rats. Thiazolidinedione, a PPAR- $\gamma$ ligand, is a common diabetic medication that can increase insulin sensitivity and ameliorate hyperglycaemia ${ }^{(21)}$. However, thiazolidinedione is still restricted in clinical use because of its side effects, such as liver toxicity ${ }^{(22)}$. PPAR- $\gamma$ is found in many tissues, and PPAR- $\gamma$ expression in skeletal muscle is important for regulating insulin sensitivity ${ }^{(23)}$ and carbohydrate metabolism ${ }^{(24)}$. In the present study, gastrocnemius muscle PPAR- $\gamma$ expression was significantly lower in the DN rats than in the control rats, and the ISI was also lower in the DN rats. A previous study has found that soya protein may stimulate PPAR- $\gamma$ expression and improve insulin sensitivity ${ }^{(25)}$. In the present study, gastrocnemius muscle PPAR- $\gamma$ expression in the $\beta$-conglycinin diet-fed rats was significantly higher than that in the DN group and the ISI was also higher than that of the DN group. The present results suggest that $\beta$-conglycinin supplementation may increase insulin sensitivity via the stimulation of gastrocnemius muscle PPAR- $\gamma$ expression. Although some studies have also reported that soya isoflavones stimulate insulin secretion in vivo and in vitro ${ }^{(26)}$, we did not find significant effects of soya isoflavones on insulin or blood sugar levels.

In the present study, rats with STZ-induced DN exhibited lower blood pressure than the control rats, which was consistent with the finding in a previous study that a STZ injection may interfere with the development of hypertension in $\mathrm{SHR}^{(27)}$. Studies have suggested that the renin-angiotensin system (RAS) in local tissues is more important for long-term blood pressure maintenance ${ }^{(28)}$ and cardiorenal remodelling ${ }^{(29)}$. Glomerular hypertension leads to increased permeability in Bowman's capsule and urinary albumin excretion, leading to the progression of $\mathrm{DN}^{(30)}$. In clinical studies, ACE inhibitors can reduce blood pressure and urinary protein excretion and retard the progression of $\mathrm{DN}^{(31,32)}$. In addition, high concentrations of Ag II in vivo may also inhibit renal nephrin expression ${ }^{(33)}$. We found that the $\beta$-conglycinin diet-fed rats exhibited lower systolic blood pressure, kidney ACE activity and Ag II concentrations. These results suggest that the ACE-inhibitory effect of $\beta$-conglycinin in the kidneys leads to a decrease in the production of Ag II and consequently plays a role in decreasing blood pressure and increasing nephrin expression. CYP4A expression in the rat kidney converts arachidonic acid to hydroxyeicosatetraenoic acid. Hydroxyeicosatetraenoic acid is a vasoactive substance that stimulates Ag II release from renal vessels and leads to an elevation in blood pressure ${ }^{(34)}$. In the present study, both $\beta$-conglycinin and soya isoflavones reduced kidney CYP4A expression, and thus, the effect of $\beta$-conglycinin on blood pressure may partially have been caused by the inhibition of CYP4A expression and the inhibitory effect of $\beta$-conglycinin on CYP4A may partially have been caused by its isoflavone content.

Hyperglycaemia increases oxidative stress and reactive oxygen species generation, inflammatory factor release ${ }^{(35)}$ and RAS activation ${ }^{(36)}$ and promotes AGE generation ${ }^{(37)}$. High blood glucose, Ag II and AGE concentrations also stimulate TGF- $\beta$ expression ${ }^{(3)}$. TGF- $\beta$ overexpression may lead to inflammation, basement membrane extracellular matrix accumulation and renal injury ${ }^{(4)}$. In the present study, $\beta$-conglycinin intervention decreased TBARS concentration and TGF- $\beta$ expression and soya isoflavones also reduced oxidative and inflammatory indicators in the DN rats. Previous studies have indicated that soya protein exhibits renal protective effects in animal models subjected to various renal diseases, which may be related to the effects of its isoflavone content, such as anti-inflammatory and antioxidative effects $^{(15)}$. For example, genistein down-regulated $T G F-\beta$ gene expression at the transcriptional level ${ }^{(38)}$. Therefore, in addition to improving hyperglycaemia and inactivating the RAS, the soya isoflavones of $\beta$-conglycinin may also normalise oxidative stress and decrease TGF- $\beta$ expression in DN rats.

Hyperlipidaemia also plays an important role in the progression of renal diseases ${ }^{(39)}$. Hyperlipidaemia may increase glomerular vascular permeability and lead to renal dysfunction. Improvement of plasma lipid profiles can retard renal failure $^{(40)}$. In the present study, the DN rats exhibited hypertriacylglycerolaemia. $\beta$-Conglycinin was reported to reduce TAG levels by decreasing sterol regulatory element-binding protein 1 (SREBP1) mRNA expression ${ }^{(12)}$ or regulating adiponectin levels ${ }^{(20)}$, and these two mechanisms are affected by insulin. Therefore, $\beta$-conglycinin can reduce plasma TAG concentrations in DN rats and may improve insulin sensitivity. Soya isoflavones may act as ligands of oestrogen receptors ${ }^{(41)}$ or regulate lipid metabolism via protein signalling ${ }^{(25)}$. In the present study, we also found that soya isoflavones lowered plasma TAG concentrations in the DN rats. The results suggest that $\beta$-conglycinin may also reduce plasma levels because of its isoflavone content.

In summary, $\beta$-conglycinin increased PPAR- $\gamma$ expression and improved insulin sensitivity and thus ameliorated hyperglycaemia and AGE generation. In addition, $\beta$-conglycinin inhibited the activity of ACE, decreased the generation of $\mathrm{Ag}$ II and retarded the elevation of blood pressure and oxidative stress. It also improved renal nephrin expression and may be beneficial for maintaining the renal filtration barrier. Although soya isoflavones can protect against oxidative stress and hyperlipidaemia in $\mathrm{DN}$, they alone were unable to retard the progression of renal dysfunction in the present study. Therefore, the beneficial effects of $\beta$-conglycinin cannot be completely explained by its isoflavone content. 


\section{Acknowledgements}

The present study was funded by the National Science Council (NSC 101-2320-B-038-016, Taipei, Taiwan). The contribution of each author was as follows: H.-Y. Y., L.-Y. W. and W.-J. Y. performed the experiments and contributed to the writing of the manuscript; J.-R. C. designed the study and was the promoter, investigator and coordinator. All authors read and approved the final contents of the manuscript. The authors declare that there are no conflicts of interest and that they have adhered to the Committee on Publication Ethics guidelines on research and publication practice.

\section{References}

1. Zimmet P, Alberti KG \& Shaw J (2001) Global and societal implications of the diabetes epidemic. Nature 414, 782-787.

2. Chukwuma C (1992) Comments on the clinical impact of hypertension in type I diabetes. $J$ Diabetes Complications 6, 197-202.

3. Ziyadeh FN \& Wolf G (2008) Pathogenesis of the podocytopathy and proteinuria in diabetic glomerulopathy. Curr Diabetes Rev 4, 39-45.

4. Dronavalli S, Duka I \& Bakris GL (2008) The pathogenesis of diabetic nephropathy. Nat Clin Pract Endocrinol Metab 4, 444-452.

5. Eid AA, Gorin Y, Fagg BM, et al. (2009) Mechanisms of podocyte injury in diabetes: role of cytochrome $\mathrm{P} 450$ and NADPH oxidases. Diabetes 58, 1201-1211.

6. Doublier S, Salvidio G, Lupia E, et al. (2003) Nephrin expression is reduced in human diabetic nephropathy: evidence for a distinct role for glycated albumin and angiotensin II. Diabetes 52, 1023-1030.

7. Liebau MC, Lang D, Bohm J, et al. (2006) Functional expression of the renin-angiotensin system in human podocytes. Am J Physiol Renal Physiol 290, F710-F719.

8. Takamatsu K, Tachibana N, Matsumoto I, et al. (2004) Soy protein functionality and nutrigenomic analysis. Biofactors 21, 49-53.

9. Banz WJ, Davis J, Peterson R, et al. (2004) Gene expression and adiposity are modified by soy protein in male Zucker diabetic fatty rats. Obes Res 12, 1907-1913.

10. Yang HY, Chen JR \& Chang LS (2008) Effects of soy protein hydrolysate on blood pressure and angiotensin-converting enzyme activity in rats with chronic renal failure. Hypertens Res 31, 957-963.

11. Kuba M, Tanaka K, Tawata S, et al. (2003) Angiotensin I-converting enzyme inhibitory peptides isolated from tofuyo fermented soybean food. Biosci Biotechnol Biochem 67, $1278-1283$.

12. Moriyama T, Kishimoto K, Nagai K, et al. (2004) Soybean beta-conglycinin diet suppresses serum triglyceride levels in normal and genetically obese mice by induction of beta-oxidation, downregulation of fatty acid synthase, and inhibition of triglyceride absorption. Biosci Biotechnol Biochem 68, 352-359.

13. Griesmacher A, Kindhauser M, Andert SE, et al. (1995) Enhanced serum levels of thiobarbituric-acid-reactive substances in diabetes mellitus. Am J Med 98, 469-475.

14. Vermeirssen V, Van Camp J \& Verstraete W (2002) Optimisation and validation of an angiotensin-converting enzyme inhibition assay for the screening of bioactive peptides. J Biochem Biophys Methods 51, 75-87.
15. Anderson JW (2008) Beneficial effects of soy protein consumption for renal function. Asia Pac J Clin Nutr 17, Suppl. 1, 324-328.

16. Mundel P \& Shankland SJ (2002) Podocyte biology and response to injury. J Am Soc Nephrol 13, 3005-3015.

17. Benigni A, Gagliardini E, Tomasoni S, et al. (2004) Selective impairment of gene expression and assembly of nephrin in human diabetic nephropathy. Kidney Int 65, 2193-2200.

18. Wolf G \& Ziyadeh FN (2007) Cellular and molecular mechanisms of proteinuria in diabetic nephropathy. Nephron Physiol 106, 26-31.

19. Davis BJ, Forbes JM, Thomas MC, et al. (2004) Superior renoprotective effects of combination therapy with ACE and AGE inhibition in the diabetic spontaneously hypertensive rat. Diabetologia 47, 89-97.

20. Tachibana N, Iwaoka Y, Hirotsuka M, et al. (2010) Betaconglycinin lowers very-low-density lipoprotein-triglyceride levels by increasing adiponectin and insulin sensitivity in rats. Biosci Biotechnol Biochem 74, 1250-1255.

21. Willson TM, Lambert MH \& Kliewer SA (2001) Peroxisome proliferator-activated receptor gamma and metabolic disease. Annu Rev Biochem 70, 341-367.

22. Lebovitz HE (2002) Differentiating members of the thiazolidinedione class: a focus on safety. Diabetes Metab Res Rev 18, Suppl. 2, S23-S29.

23. Lopez-Soriano J, Chiellini C, Maffei M, et al. (2006) Roles of skeletal muscle and peroxisome proliferator-activated receptors in the development and treatment of obesity. Endocr Rev 27, 318-329.

24. Sugita S, Kamei Y, Akaike F, et al. (2011) Increased systemic glucose tolerance with increased muscle glucose uptake in transgenic mice overexpressing RXRgamma in skeletal muscle. PLoS One 6, e20467.

25. Ronis MJ, Chen Y, Badeaux J, et al. (2009) Dietary soy protein isolate attenuates metabolic syndrome in rats via effects on PPAR, LXR, and SREBP signaling. J Nutr 139, 1431-1438.

26. Hamden K, Jaouadi B, Carreau S, et al. (2011) Therapeutic effects of soy isoflavones on alpha-amylase activity, insulin deficiency, liver-kidney function and metabolic disorders in diabetic rats. Nat Prod Res 25, 244-255.

27. Sato T, Nara Y, Kato Y, et al. (1991) Hypertensive diabetic rats: different effects of streptozotocin treatment on blood pressure in adult SHR and in neonatal SHR. Clin Exp Hypertens A 13, 981-990.

28. Gibbons GH (1998) The pathophysiology of hypertension: the importance of angiotensin II in cardiovascular remodeling. Am J Hypertens 11, 177S-181S

29. Mackenzie HS \& Brenner BM (1998) Current strategies for retarding progression of renal disease. Am J Kidney Dis $\mathbf{3 1}$, $161-170$.

30. Wolf S \& Risler T (2004) [Are all antihypertensive drugs renoprotective?]. Herz 29, 248-254.

31. Grundy SM, Benjamin IJ, Burke GL, et al. (1999) Diabetes and cardiovascular disease: a statement for healthcare professionals from the American Heart Association. Circulation 100, 1134-1146.

32. Cravedi P, Ruggenenti P \& Remuzzi G (2009) Intensified inhibition of renin-angiotensin system: a way to improve renal protection? Curr Hypertens Rep 11, 118-124.

33. Jia J, Ding G, Zhu J, et al. (2008) Angiotensin II infusion induces nephrin expression changes and podocyte apoptosis. Am J Nephrol 28, 500-507.

34. Zhang F, Qian JQ \& Wang DW (2002) Arachidonate CYP hydroxylases of kidney contribute to formation of hypertension and maintenance of blood pressure. Acta Pharmacol Sin 23, 497-502. 
35. Calcutt NA, Cooper ME, Kern TS, et al. (2009) Therapies for hyperglycaemia-induced diabetic complications: from animal models to clinical trials. Nat Rev Drug Discov 8, 417-429.

36. Ruggenenti P, Cravedi P \& Remuzzi G (2010) The RAAS in the pathogenesis and treatment of diabetic nephropathy. Nat Rev Nephrol 6, 319-330.

37. Decleves AE \& Sharma K (2010) New pharmacological treatments for improving renal outcomes in diabetes. Nat Rev Nephrol 6, 371-380.

38. Kim H, Peterson TG \& Barnes S (1998) Mechanisms of action of the soy isoflavone genistein: emerging role for its effects via transforming growth factor beta signaling pathways. Am J Clin Nutr 68, Suppl. 6, 1418S-1425S

39. Kasiske BL, Lakatua JD, Ma JZ, et al. (1998) A meta-analysis of the effects of dietary protein restriction on the rate of decline in renal function. Am J Kidney Dis 31, 954-961.

40. Kallerhoff M, Gotz L, Grone HJ, et al. (1994) Kidney preservation in situ and conservative treatment of renal cell carcinoma: application of HTK solution (CUSTODIOL). Investig Urol (Berl) 5, 116-123.

41. Bairey Merz CN, Johnson BD, Braunstein GD, et al. (2006) Phytoestrogens and lipoproteins in women. $J$ Clin Endocrinol Metab 91, 2209-2213. 